\title{
FoxO3a plays a key role in the protective effects of pomegranate peel extract against amikacin-induced ototoxicity
}

\author{
SHUANGYUE LIU ${ }^{1}$, XIAO ZHANG ${ }^{1}$, MEILING SUN ${ }^{1}$, TAO XU $^{2}$ and AIMEI WANG ${ }^{1}$ \\ ${ }^{1}$ Department of Physiology and ${ }^{2}$ Central Laboratory, Jinzhou Medical University, Jinzhou, Liaoning 121000, P.R. China
}

Received January 19, 2016; Accepted May 22, 2017

DOI: 10.3892/ijmm.2017.3003

\begin{abstract}
The use of amikacin (AMK) in present treatment strategies results in severe ototoxicity; however, the underlying molecular mechanisms of this toxicity remain unclear. In this study, we investigated the effectiveness of orally administered pomegranate peel extract (PPE), a strong antioxidant, as a protective agent against AMK-induced ototoxicity. To this end, PPE was orally administered to adult BALB/c mice for 5 days, and the mice were then concurrently treated with AMK $(500 \mathrm{mg} / \mathrm{kg} /$ day for 15 consecutive days). Auditory threshold shifts induced by AMK were significantly attenuated. The results of immunohistochemical staining and western blot analysis revealed that PPE exerted its protective effects by by downregulating the phosphorylation of Forkhead box O3a (FoxO3a), an important transcription factor which is involved in the responses to oxidative stress. The results also showed that PPE treatment inhibited mitogen-activated protein kinase phosphorylation, prevented the activation of pro-apoptotic protein caspase-3, decreased the levels of apoptosis-inducing Bax protein, and increased the levels of the anti-apoptotic mediator, Bcl-2, induced by AMK in the mouse cochlea. Taken together, our experimental findings suggest that phosphorylated FoxO3a mediates AMK-induced apoptosis in BALB/c mice cochlea. PPE effectively attenuated oxidative stress and ototoxicity by regulating FoxO3a, and may thus prove to be beneficial in protecting auditory cells from ototoxic drugs.
\end{abstract}

\section{Introduction}

Amikacin (AMK) is one of the aminoglycoside antibiotics most often used for the treatment of severe, hospital-acquired infections due to multidrug resistant Gram-negative bacteria (1). The common side-effect of AMK is ototoxicity, which results

Correspondence to: Professor Aimei Wang, Department of Physiology, Jinzhou Medical University, 40, Section 3, Songpo Road, Linghe, Jinzhou, Liaoning 121000, P.R. China

E-mail: wangaimei@Inmu.edu.cn

Key words: pomegranate peel extract, oxidative stress, forkhead box O3a, ototoxicity in hearing loss or deafness $(2,3)$. Aminoglycosides induce the generation of reactive oxygen species (ROS) and impair redox homeostasis in the inner ear (4). It has previously been demonstrated that AMK-induced ototoxicity is most likely caused by the production and accumulation of ROS, which damage the hair cells of the basilar membrane in cochlea (5).

Punica granatum L., often referred to as the pomegranate, is an edible fruit that is widely cultivated in a number of countries (6). The antioxidant effects of pomegranate peel extract (PPE) have been studied on the removal of ROS, and have been shown to inhibit lipid peroxidation in vivo (7). The antioxidant status of the cochlea has emerged as an important factor in the death or survival of hair cells with respect to 3 primary contributors that induce hearing loss: noise trauma, drugs (aminoglycoside antibiotics and cisplatin) and the aging process (8-10). A previous study indicated that oral PPE decreases the levels of ROS, as well as acute inflammation in the tympanic membrane following myringotomy (11). Moreover, the oral administration of PPE in rats has been shown to reduce cisplatin-induced toxicity in the cochlea; therefore, an oral experimental dose of PPE may have a powerful effect in protecting cisplatin-induced ototoxicity in rats (12). However, the molecular mechanisms underlying PPE-induced protection from ototoxic drugs are not yet fully understood.

Forkhead box proteins $\mathrm{O}$ (FoxO) are a subclass of the forkhead family of transcription factors, and have been linked to the regulation of oxidative stress and cellular apoptosis (13). Mammalian FoxOs consist of 4 homologous genes: FoxO1, FoxO3a, FoxO4 and FoxO6, all of which regulate various cellular functions, such as proliferation, differentiation and longevity in diverse cell types (14). ROS induce FoxO3a phosphorylation via the mitogen-activated protein kinase (MAPK) pathway, and suppress the expression of antioxidant genes, including p21, p27 and Bim, ultimately inducing cell death (15). Activated JNK is responsible for the nuclear translocation and transcriptional activity of FoxO3a (16). More importantly, FoxO3-KO mice display mild high-frequency hearing loss compared to wild-type mice (17).

In this study, we evaluated the protective effects of PPE on AMK-induced ototoxicity and the potential underlying mechanisms in vivo. We hypothesized that PPE may attenuate hearing loss and hair cell damage in response to AMK, and that these protective effects may mediated via the regulation of MAPK/ FoxO3a signaling. 


\section{Materials and methods}

Drugs and reagents. PPE was purchased from Xi'an Acetar Bio-Tech Inc. (Shaanxi, China). PPE, a brown powder that contains $\geq 98 \%$ ellagic acid (phenolic compound with antioxidant and anti-inflammatory effects), was dissolved in saline for gavage. AMK sulfate injections $(0.1 \mathrm{~g} / \mathrm{ml})$ were purchased from Qilu Pharmaceutical Co., Ltd. (Shandong, China). Rabbit polyclonal anti-FoxO3a (ab109629), anti-phospho-FoxO3a (ab47285) and anti-4-hydroxynonenal (4-HNE; ab46545) primary antibodies were from Abcam (Cambridge, UK); rabbit polyclonal anti-p38 (8690), anti-phospho-p38 (9215), anti-ERK 1/2 (4695), anti-phospho-ERK (4370), anti-JNK (9252), anti-p-JNK (9251), anti-Bcl-2 (2876), anti-Bax (5023) and anti-cleaved caspase-3 (9579) were from Cell Signaling Technology, Inc. (Danvers, MA, USA).

Animals. All animal manipulations were conducted in accordance with the regulations for the Management of Laboratory Animals published by the Ministry of Science and Technology of the People's Republic of China, and was approved by the Institutional Animal Care and Use Committee of Jinzhou Medical University. BALB/c mice (18-22 g), which were 6-8 weeks old and had normal auropalpebral reflexes and otomicroscopic examination results, were purchased from the Animal Experimental Center of Dalian Medical University [Liaoning, China; license no. SCXK (Liao) 2008-0002]. All mice were fed a standard commercial diet, and housed at an ambient temperature of $22^{\circ} \mathrm{C}$ with a relative humidity of $50 \pm 5 \%$ under $12 \mathrm{~h} / 12 \mathrm{~h}$ light-dark cycle in a specific pathogen-free facility.

Animal experiments. The experimental mice were divided into 4 groups ( $n=20$ ears in each group): i) the control group received physiological saline (100 $\mu \mathrm{l} / \mathrm{day})$ via gavage; ii) the AMK group intraperitoneally received AMK intramuscular injection at $500 \mathrm{mg} / \mathrm{kg} / \mathrm{day}$ for 15 consecutive days, as previously described (18); iii) the PPE plus AMK group not only received hypodermic injection for AMK at $500 \mathrm{mg} / \mathrm{kg} / \mathrm{day}$ for 15 consecutive days, but also PPE (34 mg/kg, $100 \mu \mathrm{l} / \mathrm{day})$ via gavage for 5 days prior to AMK injection and for 15 days concomitantly with AMK injections; and iv) the PPE group received PPE via gavage for 20 days.

Measurement of hearing function. For the analysis of the auditory threshold, the auditory brainstem response (ABR) was recorded 1 day before and 15 days after AMK treatment with tone bursts of $8,12,24$ and $32 \mathrm{kHz}$ (1-msec rise/fall time, 2-msec plateau) using the Smart EP and OAE auditory evoked potential recording system (Intelligent Hearing Systems Co., Miami, FL, USA). The mice were anesthetized using pentobarbital sodium $(40 \mathrm{mg} / \mathrm{kg}$ ) and kept warm with a heating pad during ABR recording. A subdermal (active) needle electrode was inserted at the vertex, while ground and reference electrodes were inserted subdermally in the loose skin beneath the pinnae of opposite ears. The technique used to record ABRs has been previously described in detail (19). The ABR waveforms were averaged over a 10 -msec time window using the Smart EP and OAE auditory evoked potential recording system software. The sound intensity was varied at
$5 \mathrm{~dB}$ intervals near the hearing threshold. The differences in ABR thresholds shift for each frequency between the starting and the terminal points of the experimental time course were noted. The threshold was determined off-line by two independent, experimentally blinded observers on the basis of the ABR records. Briefly, thresholds were recorded using the Smart EP and OAE auditory evoked potential recording system. In addition, the mice were euthanized and double cochleas were removed for further analysis.

Sensory hair cell counting. After the ABR test, the temporal bones were harvested. Each bulla was opened using rongeurs to expose the cochlea. The oval and round windows were then opened. Following the creation of a hole in the cochlea apex, 4\% paraformaldehyde was perfused through the cochlea for at least $24 \mathrm{~h}$. The cochlea was decalcified in 4\% EDTA for 7 days at $4^{\circ} \mathrm{C}$. Subsequently, the basilar membrane was dissected under a dissecting microscope, and the stria vascularis and tectorial membrane were removed. To identify F-actin in the organ of Corti, tetramethyl rhodamine isothiocyanate (TRITC) (Sigma-Aldrich) was applied for $20 \mathrm{~min}$ at room temperature and protected from light. The specimens were then rinsed 3 times with $0.01 \mathrm{M}$ phosphate-buffered saline (PBS) (pH 7.4). Fluorescence signals from the hair cells were counted under a BX41 microscope with epifluorescence (Olympus, Tokyo, Japan), and the images were obtained with TCS-SP5II laserscanning confocal microscope (Leica Biosystems, Wetzlar, Germany). Three rows of the outer hair cells (OHCs) were counted from the apex through the basilar turn of the cochlea under x200 magnification in 20 consecutive fields.

Immunohistofluorescence and immunohistochemical analysis. Tissue sections were incubated with rabbit anti-4-HNE antibody (1:200 dilution; Abcam), followed by appropriate fluorescent-secondary antibody for $1 \mathrm{~h}$ at room temperature. After the samples were counterstained with DAPI, immunohistofluorescence images were obtained via confocal microscopy (TCS-SP5II; Leica Biosystems). Immunofluorescence analysis was performed as previously described (19). After preparation and blocking, the sections were incubated with rabbit antiphospho-FoxO3a antibody (1:200 dilution; Abcam) overnight at $4^{\circ} \mathrm{C}$, and rinsed with TBS plus Tween-20 (TBST). The sections were incubated with biotinylated goat anti-rabbit Boost Detection reagent (Cell Signaling Technology, Inc.) for $30 \mathrm{~min}$ at room temperature, and rinsed with TBST. The chromogen reaction was performed with DAB (Cell Signaling Technology, Inc.) at room temperature, and rinsed with distilled water to terminate the reaction. The sections were observed by using Nikon microscope digital camera software (Nikon, Tokyo, Japan).

Measurement of malondialdehyde (MDA), catalase (CAT) and superoxide dismutase (SOD) activities. Cochlea tissue was homogenized in $0.01 \mathrm{M}$ cold phosphate buffer $(\mathrm{pH}$ 7.4) using a homogenizer. The homogenate was centrifuged at $1,500 \mathrm{x} \mathrm{g}$ for $10 \mathrm{~min}$. Tissue supernatant was collected and measured for oxidative stress using the MDA assay kit [2-thiobarbituric acid (TBA) method]. SOD and CAT activities were assessed using the SOD and CAT assay kit (Nanjing Jiancheng Bioengineering Institute, Nanjing, China), according to the manufacturer's instructions. 
Western blot analysis. Total cochlea tissue was homogenized in RIPA buffer (Aidlab Biotechnologies Co., Ltd, Beijing, China) containing protease inhibitor cocktail tablets and phospho-stop cocktails (cOmplete; Roche Diagnostics GmbH, Mannheim, Germany). The tissue homogenate was sonicated for $30 \mathrm{sec}$ and centrifuged at $12,000 \mathrm{rpm}$ at $4^{\circ} \mathrm{C}$ for $30 \mathrm{~min}$ to extract the supernatant. Protein concentrations were determined using the BCA kit (Thermo Fisher Scientific, Inc., San Francisco, CA, USA). The protein samples (30 $\mu \mathrm{g}$ each lane) were separated using SDS-PAGE. Following electrophoresis, the proteins were transferred onto nitrocellulose membranes (Millipore Corp., Boston, MA, USA). The membranes were then blocked in $2 \%$ BSA in TBS for $1 \mathrm{~h}$, and probed with primary antibodies rabbit anti-FoxO3a, anti-phospho-FoxO3a (1:300 dilution; Abcam), anti-p38, phospho-p38, ERK 1/2, phospho-ERK, JNK, phospho-JNK, Bcl-2, Bax and cleaved caspase-3 (1:500 dilution; Cell Signaling Technology, Inc.) overnight at $4^{\circ} \mathrm{C}$, and then washed 3 times with TBST. Subsequently, the membranes were incubated with their respective secondary antibodies $(1: 15,000$, IRDye antibodies; LI-COR Biotechnology, Lincoln, NE, USA) for 1 hour at RT. Membranes were scanned using the Odyssey infrared imaging system (LI-COR Biotechnology). The value of the relative density of each target protein band was normalized to the density of the corresponding $\beta$-actin band.

Statistical analysis. Data were analyzed using Prism 5 software (GraphPad Software, Inc., La Jolla, CA, USA), and presented as mean $\pm \mathrm{SD}$. Comparisons of parameters between different groups were performed by one-way analysis of variance (ANOVA), followed by a Newman-Keuls test. A value of $\mathrm{P}<0.05$ was considered to indicate a statistically significant difference.

\section{Results}

PPE prevents AMK-induced hearing loss and hair cell loss in mice. To verify whether PPE prevents hearing loss induced by AMK, the ABR of the experimental groups $(n=20$ ears for each group, two from each animal) was recorded. The ABR test showed that mice in the control group maintained stable thresholds throughout the experiment. By contrast, ABR threshold shifts in the AMK group were significantly elevated at 16,24 and $32 \mathrm{kHz}(25.50 \pm 3.40,45.75 \pm 9.07$ and $53.55 \pm 8.97 \mathrm{~dB}$, respectively) compared with the control group $(6.30 \pm 2.08,7.15 \pm 2.94$ and $8.00 \pm 2.73 \mathrm{~dB}$, respectively) after continuous injections for 15 days, and the hearing functional deficit was greater at the higher frequency. However, following concurrent treatment with PPE and AMK for 15 days, the ABR threshold shifts were markedly reduced compared to the AMK group at 16, 24 and $32 \mathrm{kHz}(13.95 \pm 2.98$, $30.15 \pm 3.85$ and $33.50 \pm 5.40 \mathrm{~dB})$. PPE alone had no effect on the ABR threshold shifts (Fig. 1; $\mathrm{P}<0.05$ ). To further determine the in vivo protective effects of $\mathrm{PPE}$ against $\mathrm{AMK}$, we performed TRITC staining of stereociliary bundles of hair cells and sensory hair cell counting in the 4 experimental groups. The results revealed that in the control group or PPE group alone, the morphology of the OHCs appeared normal in the lower basal turn of the cochlea. In the AMK group, OHCs had more apparent morphological changes or disorganization of stereocilia than the control group, whereas many of the OHCs in the

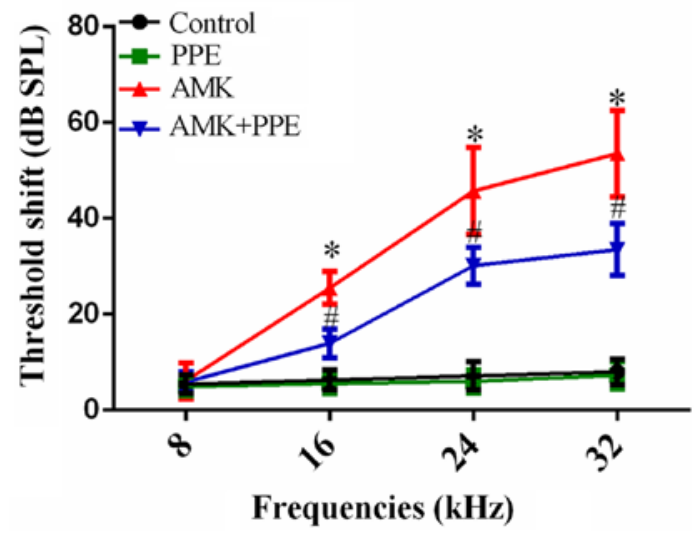

Figure 1. Pomegranate peel extract (PPE) prevents amikacin (AMK)-induced hearing loss in mouse. Auditory brainstem response (ABR) threshold shifts. Data are presented as the means $\pm \mathrm{SD}\left({ }^{*} \mathrm{P}<0.05\right.$, the AMK group vs. the control group; ${ }^{~} \mathrm{P}<0.05$, the AMK plus PPE group vs. the AMK group, $\mathrm{n}=20$ ears for each group).

AMK plus PPE group survived AMK insult (Fig. 2A). In addition, quantitative hair cell counting revealed that the amount of OHC loss was approximately $28 \%$ compared to that of approximately $58 \%$ in the AMK group without PPE (Fig. 2B; $\mathrm{P}<0.05$ ).

PPE alleviates oxidative stress in mice with AMK-induced ototoxicity. AMK signifincantly increased the staining for 4-HNE in the organ of Corti, spiral ganglion and stria vascularis of the mouse cochleae. However, the production of 4-HNE was inhibited in the mice treated with AMK plus PPE. However, PPE alone had no effect on the expression of 4-HNE in the mouse cochleae, as indicated by immunohistochemical analysis (Fig. 3A). We also observed that the AMK-treated mice had lower protein levels of CAT and SOD (the potent superoxide scavenging enzymes), and higher protein levels of MDA (a marker for oxidative stress) compared with the control mice. However, PPE administration prevented the increase in oxidative stress-related markers in the cochleae of mice treated with AMK (Fig. 3B-D; P<0.05).

PPE inhibits the AMK-induced phosphorylation of FoxO3a in mouse cochleae. Immunofluorescence staining revealed a significantly increased phosphorylation of FoxO3a in the organ of Corti, spiral ganglion and stria vascularis of the cochleae of mice treated with AMK, which was prevented to a significant degree in mice treated with AMK plus PPE (Fig. 4A and B). Similarly, the results of western blot analysis also revealed that the phosphorylation of FoxO3a in the cochleae of mice treated with AMK was increased; however PPE treatment inhibited the AMK induced the phosphorylation of FoxO3 proteins (Fig. 4C and $\mathrm{D} ; \mathrm{P}<0.05, \mathrm{n}=3)$.

PPE inhibits the activation of MAPK in cochleae of mice treated with $A M K$. The results of western blot analysis revealed the activated phosphorylation of ERK 1/2 (Thr202/Tyr204), phospho-JNK (Thr183/Tyr185) and phospho-p38 MAPK (Thr180/Tyr182), and the upregulated protein expression of JNK in the cochleae of mice treated with AMK. Of note, all these effects were inhibited in the mice treated with AMK plus PPE (Fig. 5; $\mathrm{P}<0.05$, n=3). 
A

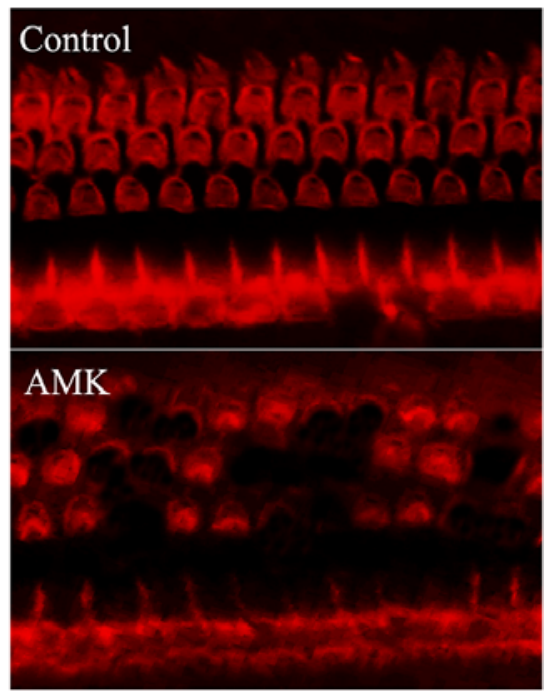

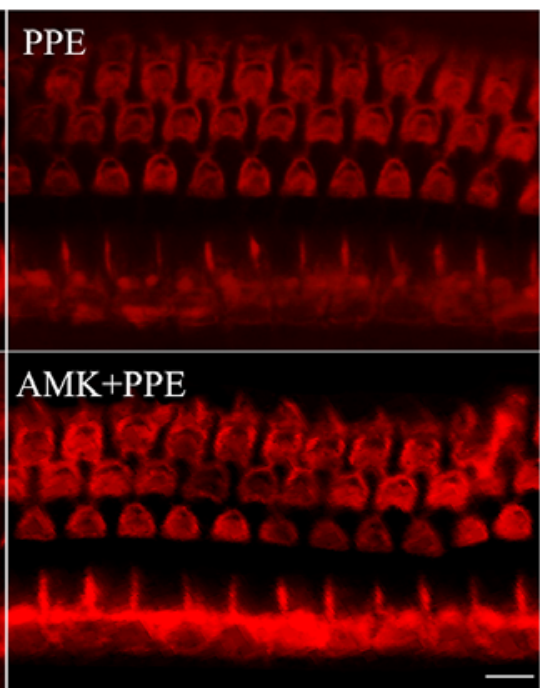

Figure 2. Pomegranate peel extract (PPE) prevents amikacin (AMK)-induced hair cell loss in mice. (A) Hair cells outlined by tetramethyl rhodamine isothiocyanate (TRITC) staining in the lower basal turn of the cochlea. Scale bar, $20 \mu \mathrm{m}$. (B) Quantitative differences of loss of outer hair cells (OHCs) from apex to basal turn of the cochlear basilar membrane (under the $\mathrm{x} 200$ microscope, 20 consecutive field) $\left({ }^{*} \mathrm{P}<0.05\right.$; $\mathrm{n}=3$ ears for each group).
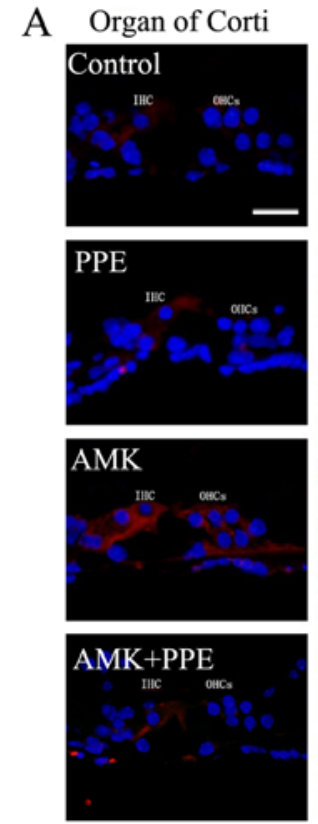

Spiral ganglion
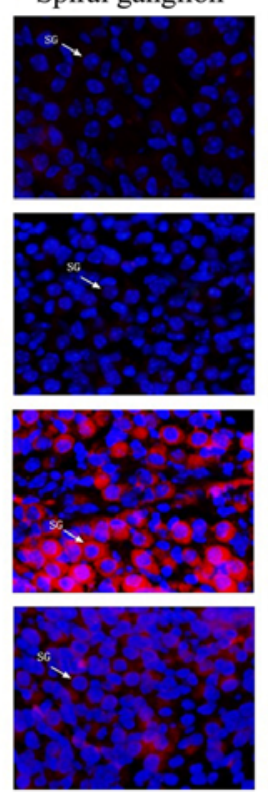

Stria
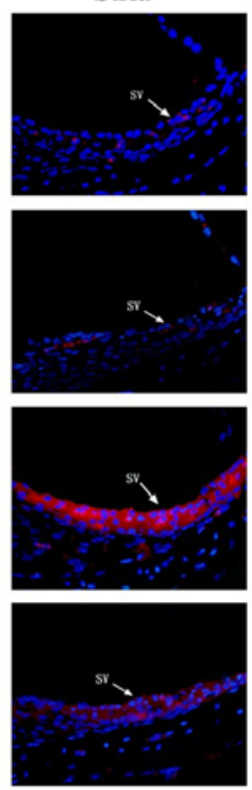

B
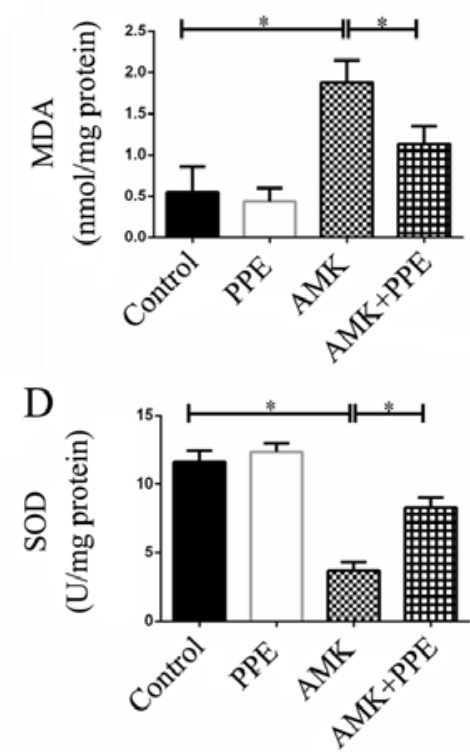

$\mathrm{C}$

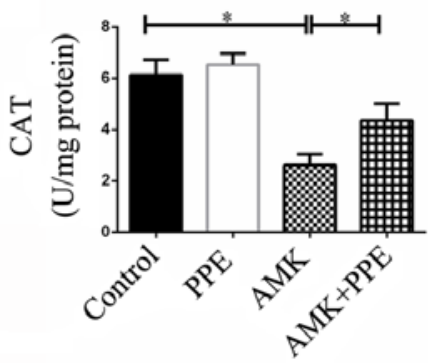

Figure 3. Pomegranate peel extract (PPE) alleviates oxidative stress in mice with amikacin (AMK)-induced ototoxicity. (A) Effect of PPE on AMK-induced expression of 4-hydroxynonenal (4-HNE) in mouse cochlea. Images of paraffin sections of the organ of Corti, spiral ganglion, and stria vascularis from BALB/c mice. The sections were labeled for 4-HNE (red) and nuclear-stained with DAPI (blue). The arrows indicate the location of 4-HNE-positive expression. White scale bar, $25 \mu \mathrm{m}$. PPE attenuated malondialdehyde (MDA) and elevated the activities of superoxide dismutase (SOD) and catalase (CAT) in cochleae of mice with AMK-induced ototoxicity. Biochemical analysis of cochlea (B) MDA, (C) CAT, and (D) SOD. Values are expressed as the means \pm SD (* $\mathrm{P}<0.05$; $\mathrm{n}=6$ ears for each group, and mean values were obtained in $\mathrm{n}=3$ independent experiments).

PPE attenuates the expression of apoptotic family proteins in the cochleae of mice treated with AMK. The results of western blot analysis revealed that the protein expression of Bcl-2 was degraded, while the expression of Bax and cleaved caspase- 3 was upregulated in the cochleae of mice in the AMK group. However, PPE inhibited the degradation of $\mathrm{Bcl}-2$ and prevented the activation of caspase- 3 in the cochleae of mice treated with AMK plus PPE (Fig. 6; $\mathrm{P}<0.05, \mathrm{n}=3$ ).

\section{Discussion}

Hair cell loss and the activation of oxidative stress may play crucial roles in the development and progression of aminoglycoside-induced ototoxicity (20). In this study, we confirmed that PPE, a crude food antioxidant, exerted protective effects against AMK-induced oxidative stress and hearing loss. In addition, the expression of phosphorylated FoxO3a was distinctly visible in the 
A Organ of Corti

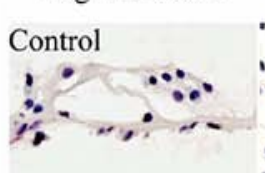

Spiral ganglion
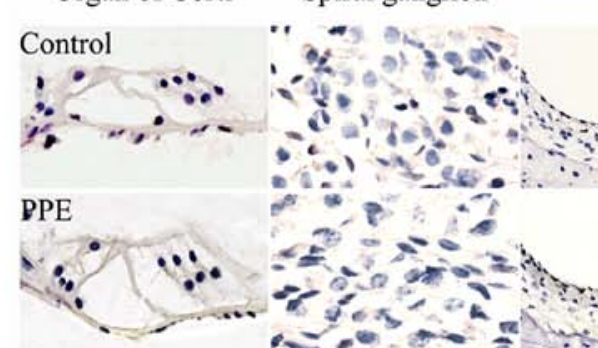

01

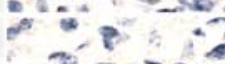

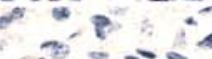

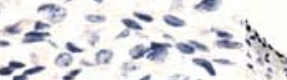

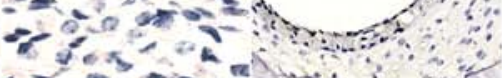

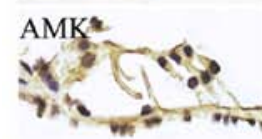

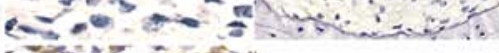
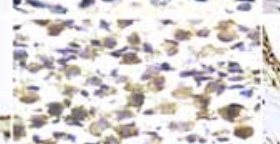

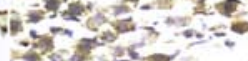

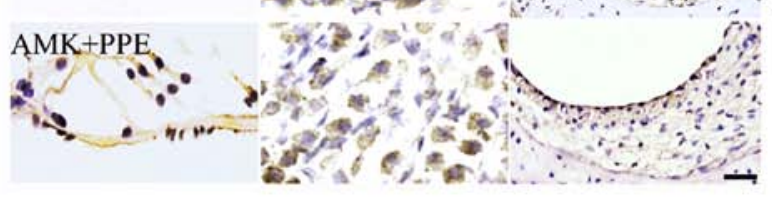

B

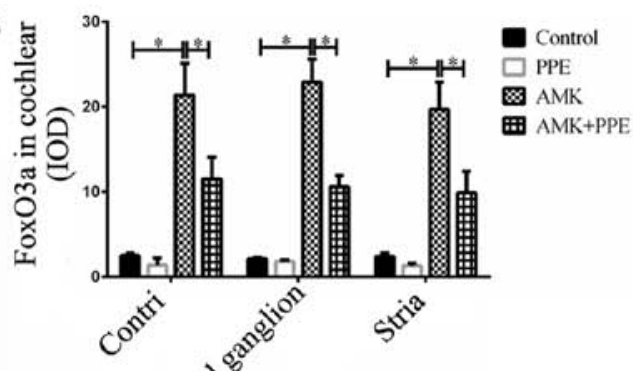

$\mathrm{C}$

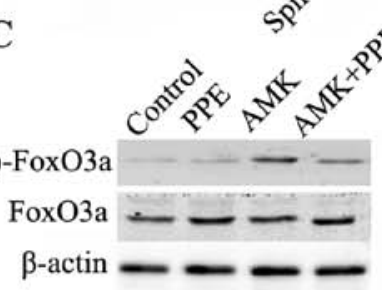

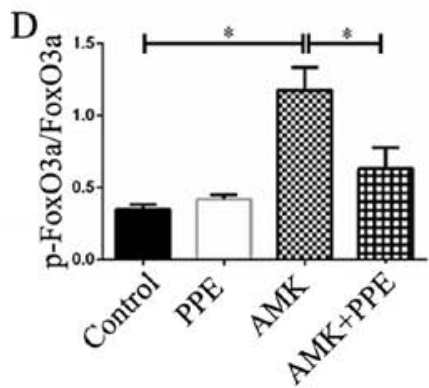

Figure 4. Pomegranate peel extract (PPE) inhibits amikacin (AMK)-induced phosphorylation of forkhead box O3a (FoxO3a) in mouse cochlea. (A) Representative immunohistochemical images of staining for phosphorylation of FoxO3a in cochlea of mice treated with AMK. Scale bar, $25 \mu \mathrm{m}$. (B) Comparison of the average integrated optical density (IOD) values for FoxO3a in 3 different sites of mouse cochlea ( ${ }^{*} \mathrm{P}<0.05 ; n=3$ ears for each group). (C and D) Western blot analysis was used to detect total and phosphorylated protein expression of FoxO3a in cochlea, with $\beta$-actin as a loading control. Data are expressed as the level of FoxO3a phosphorylation ( ${ }^{*} \mathrm{P}<0.05 ; \mathrm{n}=6$ ears for each group, and mean values were obtained in $\mathrm{n}=3$ independent experiments).

A

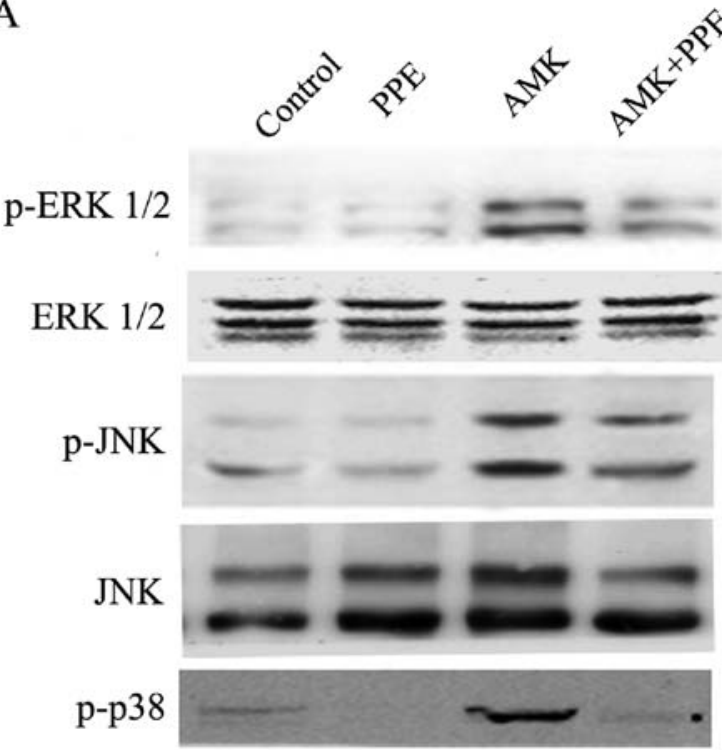

p38

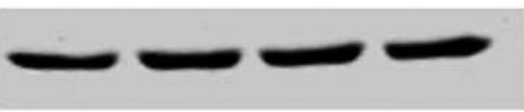

$\beta$-actin
B

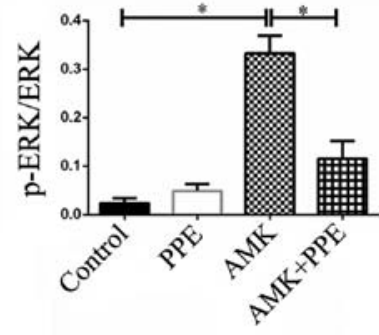

$\mathrm{C}$

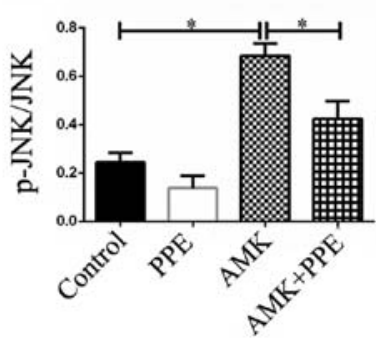

$\mathrm{D}$

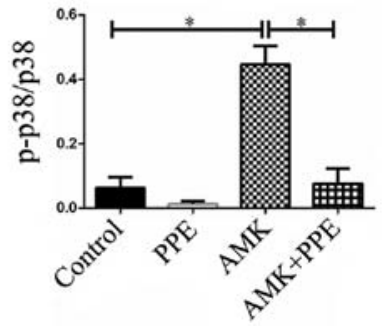

Figure 5. Pomegranate peel extract (PPE) inhibits the activation of the mitogen-activated protein kinase (MAPK) signaling pathway in cochlea of mice treated with amikacin (AMK). (A) Western blot analysis was used to quantify total and phosphorylated protein expression of ERK 1/2, JNK and p38 in cochlea, with $\beta$-actin as a loading control. Statistical analysis of the expression levels of (B) p-ERK/ERK; (C) p-JNK/JNK; and (D) p-p38/p38 ( ${ }^{*}<0.05$; $n=6$ ears for each group, and mean values were obtained in $n=3$ independent experiments).

organ of Corti, spiral ganglion and stria vascularis of the cochleae of mice with AMK-induced ototoxicity. Finally, we demonstrated that PPE inhibited AMK-induced hair cell apoptosis in the cochlea via the regulation of MAPK/FoxO3a signaling. 
A

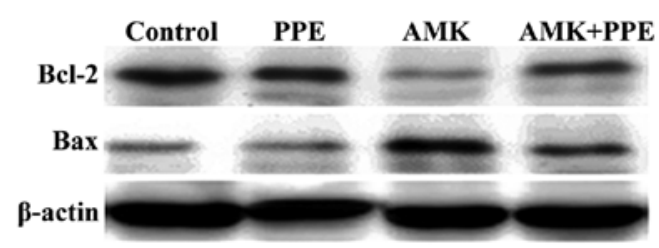

C

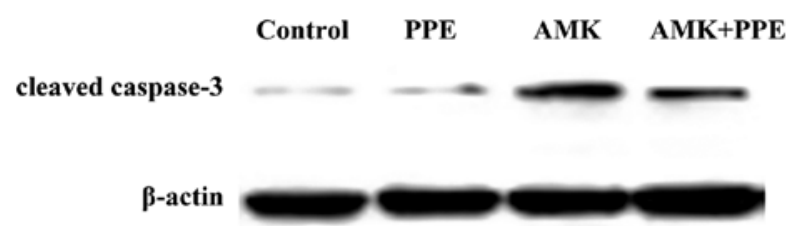

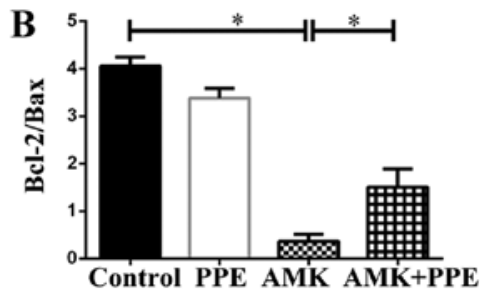

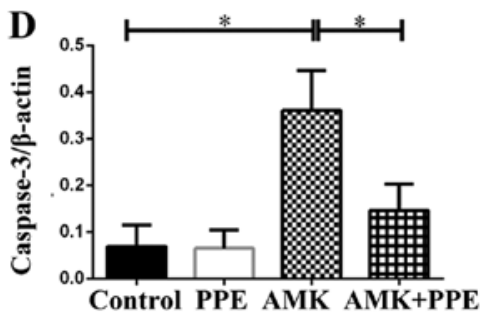

Figure 6. Pomegranate peel extract (PPE) attenuates the expression of apoptotic family proteins in cochleae of mice treated with amikacin (AMK). (A) Western blot analysis of the protein expression levels of Bcl-2 and Bax in cochlea, with $\beta$-actin as a loading control; (C) Western blot analysis of the protein expression levels of cleaved caspase- 3 , with $\beta$-actin as loading control; statistical analysis of the expression levels of $(\mathrm{B}) \mathrm{Bcl}-2 / \mathrm{Bax}$, and $(\mathrm{D}) \mathrm{caspase}-3 / \beta$-actin $\left({ }^{*} \mathrm{P}<0.05 ; \mathrm{n}=6\right.$ ears for each group, and mean values were obtained in $n=3$ independent experiments).

AMK has been shown to significantly enhance the ABR threshold shifts in guinea pigs (21). Similarly, in this study, we found that AMK-induced hearing injury in mice followed by the common characteristics of aminoglycoside ototoxicity, and more severe damage was caused at a higher frequency. A clinical study reported that the hearing thresholds of 53 hospitalized patients administered aminoglycosides were monitored at serial conventional $(0.25-8 \mathrm{kHz})$ and high-frequency $(9-20 \mathrm{kHz})$ sound stimuli. The results revealed that hearing loss occurred in $47 \%$ of the ears studied, with hearing loss first appearing in the high-frequency range in $71 \%$ of ears showing change (22). Another clinical study also showed that uremic patients with continuous ambulatory peritoneal dialysis (CAPD) peritonitis who received AMK treatment, had hearing loss, particularly at higher frequencies (2). In addition, in our study, we found hair cell deficiency from AMK ototoxicity in the lower basal turn of cochlear cells. These results also confirmed that high frequency hearing loss was mainly affected by hair cell loss at the base of the cochlea. We also found that following concurrent administration with AMK plus PPE, the ABR threshold shifts significantly decreased at 16, 24 and $32 \mathrm{kHz}$ compared with AMK, demonstrating that PPE effectively protected the mice from AMK-induced hearing injury. In addition, PPE alone had no effect on ABR threshold shifts in mice, indicating that PPE is safe and non-toxic; thus, PPE may effectively protect from aminoglycoside-induced ototoxicity.

Oxidative stress plays an important role in the pathogenesis of drug-induced ototoxicity. 4-HNE, an aldehydic product of lipid peroxidation, has been implicated in the etiology of pathological changes under oxidative stress as a key mediator of oxidative stress induced cell death (23). We observed that AMK augmented 4-HNE immunostaining in the cochlea. Furthermore, we assessed MDA, a product of lipid peroxidation, as well as two antioxidant enzymes, SOD and CAT, which are considered as biomarkers of oxidative damage (24). Our results indicated that AMK-induced ototoxicity was closely associated with elevated MDA levels, and decreased SOD and
CAT levels in cochlea. However, PPE treatment reversed the expression of AMK-induced these enzymes in the cochlea. PPE reduced ROS production to block the oxidative stress, and acted as a potential guardian against aminoglycoside ototoxicity. These results suggested that the antioxidant PPE attenuated oxidative stress, which may have beneficial therapeutic effects in the course of AMK-induced ototoxicity.

FoxO3a has recently been widely investigated as a transcription factor that is involved in regulation of the stress response, apoptosis and autophagy (25). The efficiency of FoxO3a is primarily regulated by phosphorylation. Several kinases, such as protein kinase B (Akt) and MAPK, directly phosphorylate FoxO3a. Phosphorylated FoxO3a combines with the 14-3-3 nuclear export protein and remains in the cytoplasm in cells (26). FoxO3a also plays a key role in ROS production (27) and regulation of FoxO3a by MAPK signaling in response to oxidative stress (28). In addition, FoxO3a mediates activation of antioxidant genes, such as SOD and CAT (29). A recent study showed that saponins activated FoxO3a, and nuclear factor-erythroid 2-related factor 2 (Nrf2) increased the expression and function of multiple antioxidants, at least partly contributing to the protection of D-galactose-induced aging in rats (30). In this study, we observed that AMK ototoxicity enhanced the phosphorylation of FoxO3a, and inhibited the nuclear translocation of FoxO3a in the cochlea. Our results also demonstrated that PPE inhibited the high expression of phosphorylated FoxO3a induced by AMK, which may exert beneficial effects in AMK-induced auditory insults. It has been previously reported that MAPK pathway is activated by aminoglycosides induced in rodent models, and his pathway is at least partially responsible for hair cell apoptosis (31). This may be one of the reasons that antioxidant and free radical scavengers prevent hair cell damage via MAPK dependent pathway on cochlea (32). Our group previously showed that $\alpha$-lipoic acid effectively attenuated kanamycin-induced hair cell toxicity, and was involved in MAPK activation and apoptosis in mouse hair cells (19). The present study suggested that 
PPE attenuates oxidative stress and ototoxicity by regulating MAPK/FoxO3a signaling, and the contribution of FoxO3a to the molecular mechanisms associated with AMK-induced hearing loss is a key area for future studies.

Recent studies have shown that the overexpression of Bcl-xL prevented gentamicin-induced hair cell apoptosis on the cochlea in mice $(33,34)$. In this study, we demonstrated that PPE elevated the ratio of $\mathrm{Bcl}-2 / \mathrm{Bax}$ in mice cochleae, and inhibition of cleaved caspase-3 activation led to hair cell apoptosis when treated with AMK. These results suggest that PPE may contribute to alleviated hair cell apoptosis induced by AMK.

In conclusion, our in vivo data demonstrated that PPE prevented AMK-induced ototoxicity by regulating the MAPK/ FoxO3a signaling pathway in the cochlea. Targeting FoxO3a in the cochlea may thus prove be an effective therapeutic option in drug-induced ototoxicity.

\section{Acknowledgements}

This study was funded by the Jinzhou Medical University Youth Science and Technology Staring Foundation Program (Y2012Z018), Liaoning Science and Technology Program(2014022029), Liaoning Education Program(L2015316), Undergraduate of Liaoning Province Innovation and Entrepreneurship Training Program (201410160023).

\section{References}

1. Michalopoulos A and Papadakis E: Inhaled anti-infective agents: emphasis on colistin. Infection 38: 81-88, 2010.

2. Tokgoz B, Ucar C, Kocyigit I, Somdas M, Unal A, Vural A, Sipahioglu M, Oymak O and Utas C: Protective effect of $\mathrm{N}$-acetylcysteine from drug-induced ototoxicity in uraemic patients with CAPD peritonitis. Nephrol Dial Transplant 26: 4073-4078, 2011.

3. Aksoy F, Dogan R, Ozturan O, Eren SB, Veyseller B, Pektas A and Hüseyinbas Ö: Protective effect of trimetazidine on amikacin-induced ototoxicity in rats. Int J Pediatr Otorhinolaryngol 78: 663-669, 2014.

4. Forge A and Schacht J: Aminoglycoside antibiotics. Audiol Neurootol 5: 3-22, 2000.

5. Szczepanik W, Kaczmarek P and Jezowska-Bojczuk M Oxidative activity of copper(II) complexes with aminoglycoside antibiotics as implication to the toxicity of these drugs. Bioinorg Chem Appl 2: 55-68, 2004.

6. Faria A and Calhau C: The bioactivity of pomegranate: impact on health and disease. Crit Rev Food Sci Nutr 51: 626-634, 2011

7. Al-Gubory KH, Blachier F, Faure P and Garrel C: Pomegranate peel extract decreases small intestine lipid peroxidation by enhancing activities of major antioxidant enzymes. J Sci Food Agric 96: 3462-3468, 2016.

8. Yamashita D, Jiang HY, Schacht J and Miller JM: Delayed production of free radicals following noise exposure. Brain Res 1019: 201-209, 2004.

9. Jiang H, Sha SH and Schacht J: NF-kappaB pathway protects cochlear hair cells from aminoglycoside-induced ototoxicity. J Neurosci Res 79: 644-651, 2005.

10. Jiang H, Talaska AE, Schacht J and Sha SH: Oxidative imbalance in the aging inner ear. Neurobiol Aging 28: 1605-1612, 2007.

11. Kahya V, Meric A, Yazici M, Yuksel M, Midi A and Gedikli O Antioxidant effect of pomegranate extract in reducing acute inflammation due to myringotomy. J Laryngol Otol 125: 370-375, 2011

12. Yazici ZM, Meric A, Midi A, Arınc YV, Kahya V and Hafız G: Reduction of cisplatin ototoxicity in rats by oral administration of pomegranate extract. Eur Arch Otorhinolaryngol 269: 45-52, 2012.

13. Webb AE and Brunet A: FOXO transcription factors: key regulators of cellular quality control. Trends Biochem Sci 39 159-169, 2014
14. Almeida M: Unraveling the role of FoxOs in bone - insights from mouse models. Bone 49: 319-327, 2011.

15. Parody JP, Ceballos MP, Quiroga AD, Frances DE, Carnovale CE Pisani GB, Alvarez ML and Carrillo MC: FoxO3a modulation and promotion of apoptosis by interferon- $\alpha 2 \mathrm{~b}$ in rat preneoplastic liver. Liver Int 34: 1566-1577, 2014.

16. Shukla S, Rizvi F, Raisuddin S and Kakkar P: FoxO proteins' nuclear retention and $\mathrm{BH} 3$-only protein Bim induction evoke mitochondrial dysfunction-mediated apoptosis in berberine-treated HepG2 cells. Free Radic Biol Med 76: 185-199, 2014.

17. Gilels F, Paquette ST, Zhang J, Rahman I and White PM: Mutation of Foxo3 causes adult onset auditory neuropathy and alters cochlear synapse architecture in mice. J Neurosci 33: 18409-18424, 2013.

18. Murillo-Cuesta S, Contreras J, Cediel R and Varela-Nieto I: Comparison of different aminoglycoside antibiotic treatments to refine ototoxicity studies in adult mice. Lab Anim 44: 124-131, 2010.

19. Wang A, Hou N, Bao D, Liu S and Xu T: Mechanism of alpha-lipoic acid in attenuating kanamycin-induced ototoxicity. Neural Regen Res 7: 2793-2800, 2012.

20. Shulman E, Belakhov V, Wei G, Kendall A, Meyron-Holtz EG, Ben-Shachar D, Schacht J and Baasov T: Designer aminoglycosides that selectively inhibit cytoplasmic rather than mitochondrial ribosomes show decreased ototoxicity: a strategy for the treatment of genetic diseases. J Biol Chem 289: 2318-2330, 2014.

21. Amora LdeA, Murashima AdeA, Rossato M, Moreira MB, Hyppolito MÂ and Fagundes DJ: The effects of hyperbaric oxygen therapy upon ototoxic injuries produced by amikacin in guinea pigs. Braz J Otorhinolaryngol 79: 342-348, 2013.

22. Fausti SA, Henry JA, Schaffer HI, Olson DJ, Frey RH and McDonald WJ: High-frequency audiometric monitoring for early detection of aminoglycoside ototoxicity. J Infect Dis 165: 1026-1032, 1992

23. Choi CH, Chen K, Du X, Floyd RA and Kopke RD: Effects of delayed and extended antioxidant treatment on acute acoustic trauma. Free Radic Res 45: 1162-1172, 2011.

24. Khalil SR, Abd-Elhakim YM, Selim ME and Al-Ayadhi LY: Apitoxin protects rat pups brain from propionic acid-induced oxidative stress: the expression pattern of Bcl-2 and caspase- 3 apoptotic genes. Neurotoxicology 49: 121-131, 2015.

25. Storz P: Forkhead homeobox type O transcription factors in the responses to oxidative stress. Antioxid Redox Signal 14: 593-605, 2011.

26. Vogt PK, Jiang H and Aoki M: Triple layer control: phosphorylation, acetylation and ubiquitination of FOXO proteins. Cell Cycle 4: 908-913, 2005

27. Zhang L, Yang X, Li X, Li C, Zhao L, Zhou Y and Hou H: Butein sensitizes HeLa cells to cisplatin through the AKT and ERK/p38 MAPK pathways by targeting FoxO3a. Int $\mathbf{J}$ Mol Med 36: 957-966, 2015.

28. Sun L, Zang WJ, Wang H, Zhao M, Yu XJ, He X, Miao Y and Zhou J: Acetylcholine promotes ROS detoxification against hypoxia/reoxygenation-induced oxidative stress through FoxO3a/PGC-1 $\alpha$ dependent superoxide dismutase. Cell Physiol Biochem 34: 1614-1625, 2014.

29. Rangarajan P, Karthikeyan A, Lu J, Ling EA and Dheen ST: Sirtuin 3 regulates Foxo3a-mediated antioxidant pathway in microglia. Neuroscience 311: 398-414, 2015.

30. Li YN, Guo Y, Xi MM, Yang P, Zhou XY, Yin S, Hai CX, Li JG and Qin XJ: Saponins from Aralia taibaiensis attenuate D-galactose-induced aging in rats by activating FOXO3a and Nrf2 pathways. Oxid Med Cell Longev 2014: 320513, 2014.

31. Yang J, Wu Z, Renier N, Simon DJ, Uryu K, Park DS, Greer PA, Tournier C, Davis RJ and Tessier-Lavigne M: Pathological axonal death through a MAPK cascade that triggers a local energy deficit. Cell 160: 161-176, 2015.

32. Dinh CT, Goncalves S, Bas E, Van De Water TR and Zine A: Molecular regulation of auditory hair cell death and approaches to protect sensory receptor cells and/or stimulate repair following acoustic trauma. Front Cell Neurosci 9: 96, 2015.

33. Dong Y, Liu D, Hu Y and Ma X: NaHS protects cochlear hair cells from gentamicin-induced ototoxicity by inhibiting the mitochondrial apoptosis pathway. PLoS One 10: e0136051, 2015.

34. Chi NU, Tabuchi K, Nakamagoe M, Nakayama M, Nishimura B and Hara A: Ceramide/sphingomyelin cycle involvement in gentamicin-induced cochlear hair cell death. Arch Toxicol 89: 415-421, 2015. 